\title{
Securing cartilage grafts in closed rhinoplasty
}

\begin{abstract}
Contour defects of the nose, in secondary cases or if encountered during primary rhinoplasty, are corrected by cartilage grafts in the majority of cases. Securing these grafts is tricky and time consuming, especially in closed rhinoplasty. There is still wide debate regarding the need for open or closed rhinoplasty which is very surgeon specific. Open rhinoplasty proponents use the difficulties in securing cartilage grafts as an example of one of the disadvantages to the closed approach. We describe a simple and quick suture method of securing cartilage grafts externally, to correct a range of defects in closed rhinoplasty, for which we have seen no disadvantage.
\end{abstract}

This work was presented at The $39^{\text {th }}$ Annual Meeting of The Israel Society of Plastic $\&$ Aesthetic. This research received no specific grant from any funding agency in the public, commercial, or not-for-profit sectors.
Volume 3 Issue 2 - 2019

\author{
Filson S,' Moscona R² \\ 'Department Plastic Surgery, Rambam Health Care Campus, \\ Israel
}

${ }^{2}$ Departments of Plastic Surgery, Italian Hospital, Israel

Correspondence: Simon A Filson, Consultant, Department Plastic Surgery, Rambam Health Care Campus, Ha Aliya Ha Shniya St 8 Haifa, 3 I0960I, Israel,

Emailsimonfilson83@gmail.com

Received: June II, 2017 | Published: April 02, 2019

Keywords: rhinoplasty closed, primary, secondary, defect, dorsal, cartilage grafting cosmetic

\section{Introduction}

Nasal contour deformities, like over-correction of the dorsum in primary rhinoplasty (after humpectomy) or over correction of tip/ other cartilages, can lead to aesthetically poor results. The revision of which will require a whole range of complex techniques in a secondary procedure. The use of cartilage grafts is part of the basic armamentarium in any rhinoplasty, but if used properly at the end of primary rhinoplasty it can prevent future revisional surgery. Meticulous placement of these grafts is of crucial importance. Most plastic surgeons, especially those who favor the open approach, like Rohich, ${ }^{1}$ will secure these grafts by internal multiple sutures. However, if the closed approach is used, securing these grafts becomes a major issue and time consuming. ${ }^{2-9}$ For many years, the experienced senior author of this paper has made use of already excised nasal cartilages, in order to fill different contour defect that are sometimes found at the end of surgery. These grafts are simply placed and secured externally, with a suture through the skin. The long term results of this simple technique in augmentation, and so camouflage of the contour defect, are impressive.

\section{Technique}

In all rhinoplasty, after excision, the upper lateral cartilages are stored in sterile wet gauze. If on-table assessment, at the end of primary rhinoplasty, has shown a contour defect (Figure $1 \& 2$ ), the stored upper lateral cartilage (or other residual septal cartilage) is crushed in a morcelising clamp (Figure 3) and shaped to the defect (Figure 4). This is then externally secured with a 4/0 Vicryl Rapide (what the author uses to close the nasal incisions anyhow) (Figures $5 \& 6$ ). The suture(s) is secured loosely to allow for the swelling of the soft tissue (as to not "cheesewire" the skin). Some surgeons use bolster-type dressings in order to attempt to protect the skin from direct pressure. We have not found a bolster to be necessary and have found no evidence of scarring or pressure from the simple interrupted suture. We do however see disadvantages in the use of a bolster. Not only is it unnecessarily time consuming, every rhinoplasty is taped and then casted, which would be problematic with a bolster in situ. After the suture, the external splint is then placed as standard. A small line is inscribed with a marker pen on the splint (Figure 7), in order to draw attention to the person who removes the splint to be more cautious in this area. One week later the splint and suture(s) are removed in outpatient clinic.

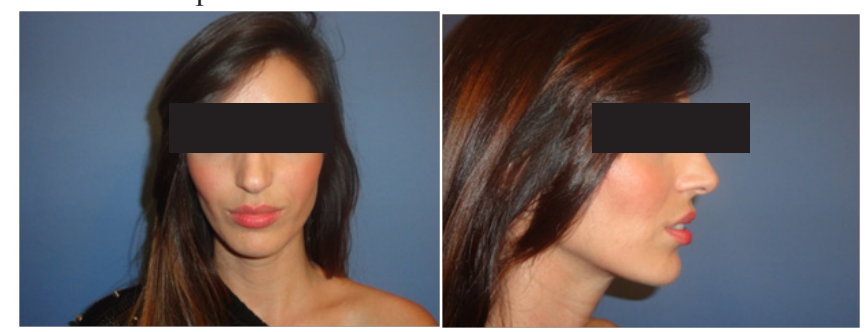

Figure I Pre Operative photos

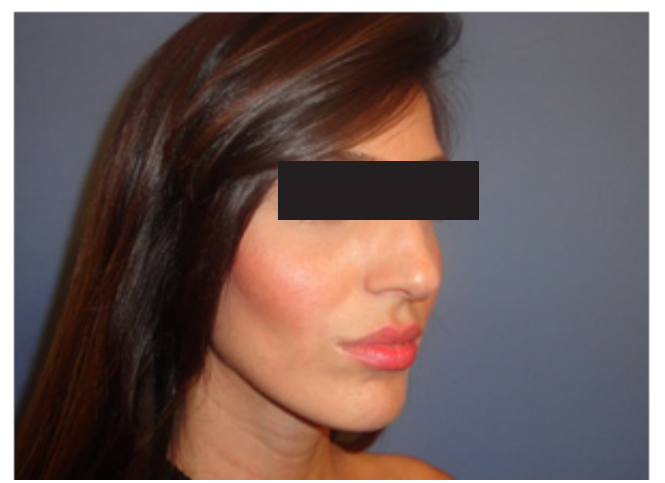

Figure 2 Excessive hump resection in primary closed rhinoplasty

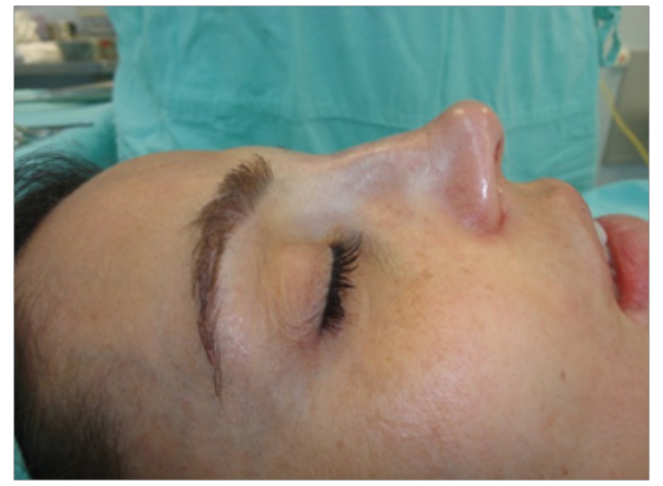

Figure 3 Crushing of cartilage in morcelising clamp 


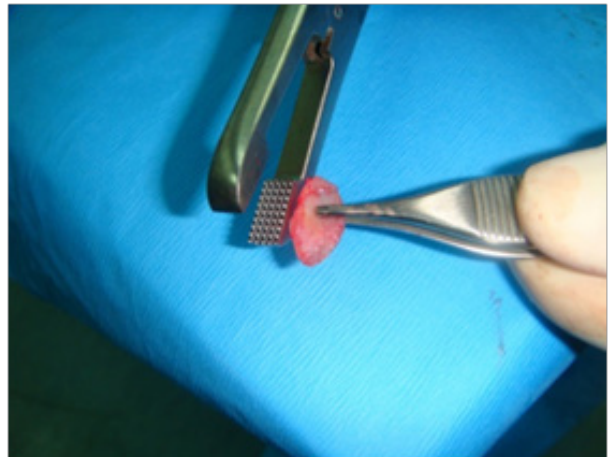

Figure 4 Shaping of the Cartilage

\section{Discussion}

In view of the ongoing debate between closed and open rhinoplasty advocates we thought there is a need to bring to light a very simple method of securing grafts in primary and secondary rhinoplasty, performed by the closed approach. The use of cartilage grafts is very common (mainly in open rhinoplasty) even though lately there is considerable doubt about their long term effectiveness. ${ }^{1}$ This simple interrupted percutaneous suture is quick and easy to perform and secures the cartilage graft in exactly the position the surgeon requires. As the suture is tied loosely and removed within 7days, we have not seen any evidence of scarring, contour defects or "train tracks" in over 30 years of the senior authors practice.

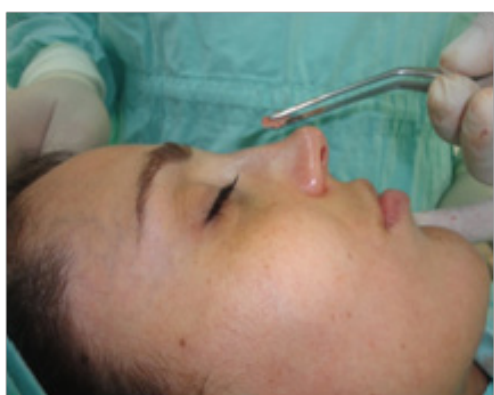

Figures 5 \& 6 Accurate external control of position and placement of securing suture through the skin

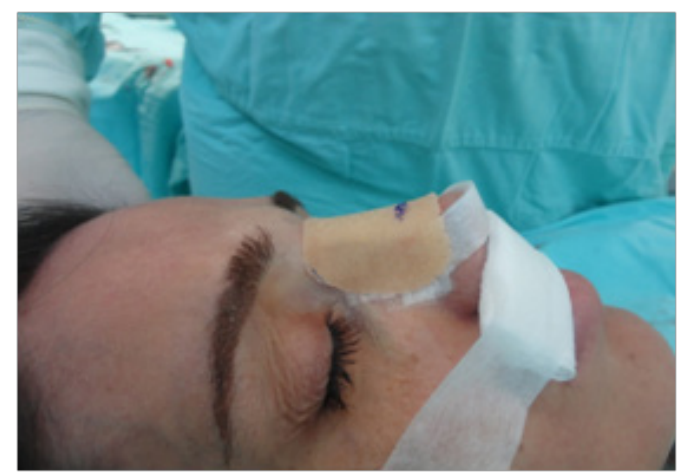

Figure 7 Splint Placement with marking to show suture

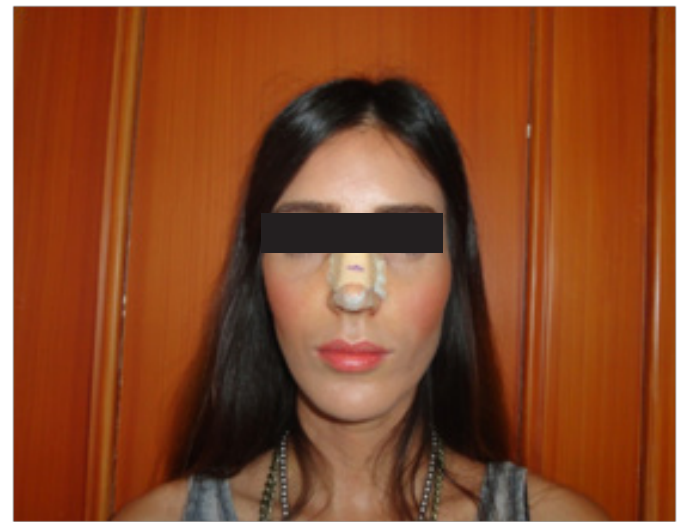

Figure 8 Week one post operative

From a literature review, only one paper in $200^{10}$ has mentioned an almost similar method of securing cartilage grafts in rhinoplasty. The author uses multiple cartilage grafts (up to 4per case) in primary rhinoplasty, almost as a routine. We differ, as we only use grafts only when needed, mainly to correct defects that would otherwise require secondary rhinoplasty correction. The paper also describes the use of a horizontal mattress suture, which we find excessive, as a simple interrupted suture works the same, without the increased chance of tissue necrosis and scarring.
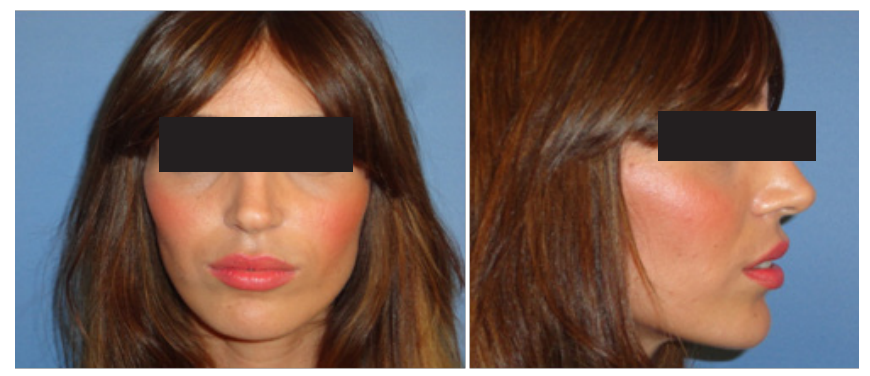

Figure 93 month post operative

\section{Conclusion}

We present a simple and quick method of securing cartilage grafts. As can be seen by the results, this easy to perform technique is very efficient at correcting contour defects in closed rhinoplasty, without the need for complex and time consuming techniques.

There are many substances that help to rebuild harmonically" cartilage grafts, especially when endogenous, in post surgical reconstruction. Some of these, sylimarine or spilanthol gattefossè could be exceptional to permit cartilage grafts can remain safe and never change colour and dermal consistency remains the same perennially. This is only in order to avoid the characteristic of being a cosmetic camouflage in such an important concern (That involves touch ups in the future).

\section{Acknowledgments}

None. 


\section{Conflict of interest statement}

All the authors have no financial or personal relationships with other people or organizations that could inappropriately influence or bias their work.

\section{References}

1. Rohrich RJ. Rhinoplasty - Getting It Right the First Time.

2. Neto JC, Fernandez DE, da Silva Carvalho G. Tip Grafts in Closed Rhinoplasty: Insertion and Fixation Made Easy. Plast Reconstr Surg. 2008;121(3):126e.

3. Lanfranchi LA, Gazzola, R, Addis A, et al. An inexpensive and easy technique for placing and fixing dorsal grafts in rhinoplasty. Plast Reconstr Surg. 2010;126(6):311e-312e.

4. Oneal RM, Berkowitz RL. Upper lateral cartilage spreader flaps in rhinoplasty. Aesthet Surg J.1998;18(5):370-371.
5. Gruber RP, Park E, Newman J, et al. The spreader flap in primary rhinoplasty. Plast Reconstr Surg. 2007;119(6):1903-1910.

6. Ingels KJ, Orhan KS, Heerbeek N. The effect of spreader grafts on nasal dorsal width in patients with nasal valve insufficiency. Arch Facial Plast Surg. 2008;10(5):354-356.

7. Faris C, Koury E, Kothari P, et al. Functional rhinoplasty with batten and spreader grafts for correction of internal nasal valve incompetence. Rhinology. 2006;44(2):114-117.

8. Byrd HS, Meade RA, Gonyon DL. Using the autospreader flap in primary rhinoplasty. Plast Reconstr Surg. 2007;119(6):1897-1902.

9. Cutting CB. Cerclage suture method for closed-tip rhinoplasty. Plast Reconstr Surg. 1999;104(6):1839-1842.

10. Karen M, Lian T, Stucker F. The use of percutaneous sutures for graft fixation in rhinoplasty. Arch Facial Plast Surg. 2003;5(2):193-196. 